\title{
Non-cardiovascular findings on chest CT angiography in children with congenital heart disease: How important are they?
}

\author{
Yaotse Elikplim Nordjoe ${ }^{*}$, Suzanne Rita Aubin Igombe ${ }^{\dagger}$ and Latifa Chat ${ }^{\dagger}$
}

\begin{abstract}
Background: There are only a few publications about the non-cardiovascular findings in children with congenital heart diseases explored by chest CT angiography. The purpose of our study is to evaluate the prevalence of non-cardiovascular findings on chest CT angiographies in children with congenital heart disease and to raise awareness about their importance among the radiologists.

Methods: We retrospectively reviewed the 272 chest $C T$ angiographies performed in our pediatric radiology department between January 2017 and march 2021 and extracted the data of the 180 patients positive for a congenital heart disease. Then from that pool, we sorted out the non-cardiovascular findings into significant and non-significant in regard of their relevance or not in the patient's management.

Results: Non-cardiovascular lesions were found in 58\% (105/180) of our patients, and 49\% (88/180) of them presented at least one significant non-cardiac lesion. Lung and airways abnormalities were found in $41 \%(74 / 180)$ of the cases, representing the majority of the non-cardiovascular findings. Syndromic associations were found in 17\% (28/180) of our patients, including 14 cases of heterotaxic syndrome.

Conclusion: Non-cardiovascular findings are common in children with congenital heart disease. Reporting these associated lesions is a requisite for an optimal therapeutic management of these children. Radiologists should never forget that a Chest CT angiography in children is first and foremost a chest CT.
\end{abstract}

Keywords: Congenital heart disease, Chest CT angiography, Children, Non-cardiovascular findings

\section{Background}

The congenital heart diseases are relatively common and very diverse, with an estimated prevalence of 1-4 per 1000 births [1-3]. In children, in order to align with the ALARA "As Low As Reasonably Achievable" principle, cardiac ultrasound and MRI represent excellent imaging tools for diagnosing and monitoring patients with

\footnotetext{
${ }^{*}$ Correspondence: nordmichel2002@yahoo.fr

${ }^{\dagger}$ Yaotse Elikplim Nordjoe, Suzanne Rita Aubin Igombe and Latifa Chat equally contributed to this work.

Pediatric Radiology Department, Children's Hospital, Centre HospitaloUniversiataire Ibn Sina, Rue Lamfadel Cherkaoui Rabat - Institut, B.P 6527 , Rabat, Morocco
}

congenital heart disease $[4,5]$. However, they are not perfect and not suitable for all situations.

With the development of multi-detector scanners, dose reduction techniques, better reconstruction algorithms and global advances in the field of computer science, chest CT angiography has become and accepted as a rapid and non-invasive diagnosing and monitoring tool in children with congenital heart disease [4, 6-11].

CT allows a detailed regional analysis and therefore, enables the detection of findings other than cardiovascular. These non-cardiovascular findings can have a major impact in the management of the patient. Their prevalence and implications have been the subject of 
numerous publications in adult cardiac pathology with a highly variable prevalence ranging from 7.1 to $81 \%$ [1219]. On the contrary, in the pediatric population, there are only three publications studying non-cardiovascular findings in congenital heart disease, two on CT angiography $[20,21]$ and the other on MR angiography [22].

The aims of our work are to evaluate the prevalence and relevance of non-cardiovascular findings on chest $\mathrm{CT}$ angiography in children with congenital heart disease in our institution and to raise awareness among the radiologists about the importance of their detection.

\section{Material and methods}

\section{Description of the study}

This is a retrospective observational study over a period of 4 years and 3 months (between January 2017 and march 2021). Analysis was performed on the database of the radiology department of the children's hospital in Rabat-Morocco. We reviewed all the 272 chest CT angiographies performed in the above-mentioned period, for a known or suspected congenital heart disease, of which the 180 patients positive for a congenital heart disease formed the pool of our study. From that pool, non-cardiovascular findings have been identified and processed to evaluate their prevalence and their relevance.

Patients had to be less than 18 years old.

Since the study is based on a retrospective analyze of our archiving database, the obtention of a written informed consent was waived by our institution ethical committee.

\section{CT angiography procedure Preparation of the patients}

A reassurance of the patient was achieved by the parents and the medical staff. If needed, one of the parents was authorized-after protection-to remain in the machine room with the child. A peripheral intravenous access was obtained using a 20 to 24 Gauge catheter placed in an antecubital forearm or hand vein. A little sedation was sometimes necessary; performed by the anesthesiologist assigned to the scanner.

\section{CT examination protocol}

All the exams were performed on a single-source 16-channel MDCT scanner (SIEMENS Somatom Emotion 16).

An integrated pre-set chest CT angiography protocol was used, allowing the adaptation of the acquisition parameters to the pediatric population: $80-100 \mathrm{kV}$, with for example $30 \mathrm{mAs}$ at $3 \mathrm{~kg}$ and $40 \mathrm{mAs}$ at $5 \mathrm{~kg}$. All the examinations were non-electrocardiogram-gated and were acquired during shallow free-breathing or suspended inspiration. Contrast medium was power injected
(Medrad; Stellant), using 1-2 $\mathrm{ml} / \mathrm{kg}$ of nonionic contrast medium (Ultravist $300 \mathrm{mg} \mathrm{I} / \mathrm{ml}$; Bayer Schering Pharma) at a rate of $0.5-3.0 \mathrm{ml} / \mathrm{s}$, followed by $10-30 \mathrm{ml}$ of saline at a similar rate. Automated bolus tracking was used to achieve optimal opacification. Data sets were reconstructed into 1 to $1.5 \mathrm{~mm}$ contiguous slices. We only used filtered back projection algorithm for the reconstructions. Reconstructed slice thickness was $1.5 \mathrm{~mm}$ with H30 kernel for mediastinum and $1 \mathrm{~mm}$ with H80 kernel for lung. The mean DLP was $45.56 \mathrm{mGy} / \mathrm{cm}( \pm 23.81)$. The mean CTDIv was $1.57 \mathrm{mGy}( \pm 0.52)$.

\section{Definition of non-cardiovascular findings}

Non-cardiovascular findings are abnormalities other than those of the heart and vessels, discovered on the volume acquired.

A non-cardiovascular finding was "significant" if further investigations were needed and/or if this abnormality contributed to the worsening of the patient's clinical state.

A "significant" finding could either be "expected" or "unexpected". An "expected" lesion is one whose occurrence is not surprising, being part of the usual associated lesions of that particular congenital heart disease while an "unexpected" lesion is just the opposite of that.

It is important to note that the same finding can be significant in one patient but non-significant in another one, depending on its impact on the patient's condition. Also, there are some findings whose categorization cannot be formally certain because the physio-pathological implications are complex.

\section{Data collection and analysis}

Post processing and analysis were carried out on a dedicated post processing workstation with SyngoVia software, by a certified senior radiologist with 10 years of experience in congenital heart diseases (Dr L.C.).

The entire arsenal of this platform was used, allowing both usual multiplanar reformatting and MIP, MInIP, VRT 3D reconstructions etc.

Images and reports were archived on our picture archiving and communication systems VISIONPACS and VISIONRIS.

The retrospective analysis of the data was carried out on these above-mentioned archiving platforms. First, all CT scans performed for the indication of known or suspected congenital heart disease were extracted. A second sorting allowed the separation of the normal examinations from the examinations on which there was indeed a congenital heart disease. Lastly, the pool of congenital heart disease was analyzed to deduce those with associated non-cardiovascular findings. These non-cardiovascular findings were subcategorized into: infectious, 
malformative, tumoral lesions and others. Also, an anatomical distribution of these abnormalities was made, into supra-diaphragmatic findings (pulmonary and airways, mediastinal) and infra-diaphragmatic findings (liver, kidney, spleen, bowel). Bone skeletal abnormalities have also been isolated and finally, syndromic associations were identified.

\section{Results}

\section{Overall results}

A total of 272 chest $\mathrm{CT}$ angiographies was performed for a known or suspected congenital heart disease, of which, 180 was positive for a congenital heart disease. The mean age was 3.1 years $( \pm 3.2)$, ranging from 1 day to 17 years. Some of our patients $(n=20)$ had a history of surgery (Table 1).

We detected 155 non-cardiovascular anomalies in 105 patients positive for congenital heart disease $(58 \%$ of 180), of which 88 patients had significant anomalies and 17 patients had non-significant anomalies. More than one non-cardiovascular anomalies were found in 24 patients (23\% of 105$)$. In these cases where there were association of several abnormalities in the same patient, if there is only one significant finding; the patient was classified as significant (Table 2).

Non-cardiovascular findings were classified by subcategories: infectious, malformative, tumoral and others (Fig. 1).
Table 2 Distribution of significant and non-significant findings

\begin{tabular}{lc}
\hline Non-cardiovascular findings & $\begin{array}{l}\mathbf{n}=\text { Patients } \\
\text { (\% of 180) }\end{array}$ \\
\hline Significant & $88(49 \%)$ \\
Expected & $66(37 \%)$ \\
Unexpected & $22(12 \%)$ \\
Non-significant & $17(9 \%)$ \\
Total & $105(58 \%)$ \\
\hline
\end{tabular}

Topographic distribution and syndromic associations The non-cardiovascular findings were subdivided into four main groups: supra diaphragmatic, infra diaphragmatic, skeletal and syndromic associations.

\section{Supra-diaphragmatic anomalies (Table 3)}

Two subsets were isolated. On the one hand lung and airways findings were identified in $74(41 \%$ of the 180 patients of our series) patients and on the other, mediastinal findings were identified in 3 patients. Lung and airways findings were by far the most frequently encountered anomalies, mainly dominated by infectious pneumopathies, mosaic attenuation pattern and atelectasis. Associations can be found in the same patient.

Table 1 Characteristics of the study population

\begin{tabular}{|c|c|c|}
\hline Population with congenital heart disease & Mean age $\pm S D$ & Range \\
\hline $\mathrm{n}=180(99 \mathrm{M} / 81 \mathrm{~F})$ & 3,1 years $\pm 3,2$ & 1 day-17 years \\
\hline Patients with or without non-cardiovascular findings & & $\mathbf{n}=$ patients \\
\hline Patients with non-cardiovascular finding & & $105(58 \%$ of 180$)$ \\
\hline Patient without non-cardiovascular findings & & 75 (42\% of 180$)$ \\
\hline History of surgical procedure & & $\mathrm{n}=$ patients \\
\hline Blalock-Taussig-Thomas & & 11 \\
\hline Coarctation repair & & 3 \\
\hline Fontan & & 1 \\
\hline Pulmonary artery prosthesis & & 1 \\
\hline Tetralogy of Fallot repair & & 3 \\
\hline Senning & & 1 \\
\hline Repartition by congenital heart disease & & $\mathrm{n}=$ patients \\
\hline Left obstructive lesions & & 42 \\
\hline Right obstructive lesions & & 33 \\
\hline Cono-truncus anomalies & & 30 \\
\hline Congenital arch anomalies & & 30 \\
\hline Septation anomalies & & 35 \\
\hline Pulmonary venous anomalies & & 10 \\
\hline
\end{tabular}




\section{NON CARDIOVASCULAR FINDINGS BY THEME}

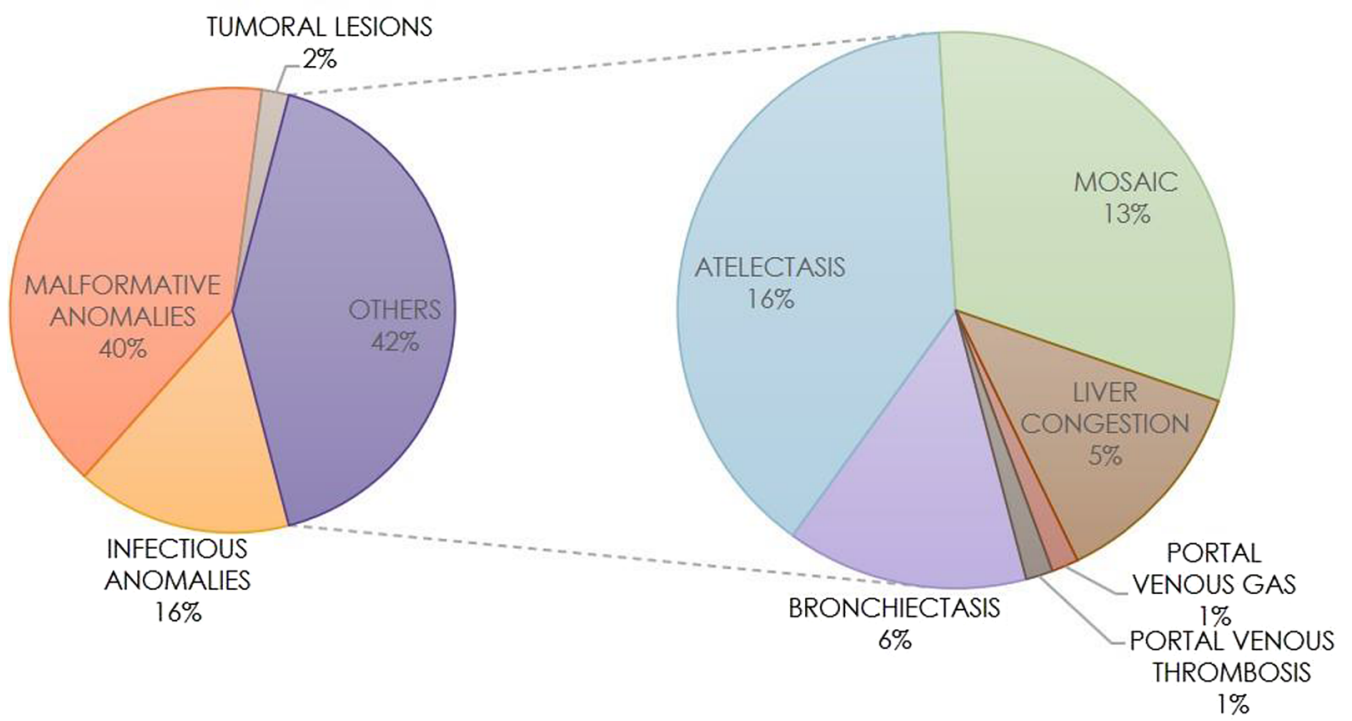

Fig. 1 Nature of the non-cardiovascular findings. Distribution of the 155 non-cardiovascular findings detected in the 105 patients positive for congenital heart disease (58\% of 180)

Table 3 Distribution of supra-diaphragmatic findings

\begin{tabular}{llc}
\hline Localization & Anomalies & $\mathbf{n}=$ Patients \\
\hline Lung and airways & Pulmonary hypoplasia & 5 \\
& Pneumonia & 23 \\
& Bronchiectasis & 9 \\
& Atelectasis & 25 \\
& Mosaic attenuation pattern & 20 \\
& Emphysema & 8 \\
& Pulmonary sequestration & 3 \\
\cline { 2 - 2 } & Vascular compression of the trachea & 3 \\
& Pericardial effusion & 1 \\
& Thymic agenesis & 2 \\
\hline
\end{tabular}

Infra-diaphragmatic anomalies (Table 4, Figs. 2, 3)

Four subsets were isolated: Liver, kidney, spleen and bowel. Splenic anomalies were essentially asplenia or polysplenia mainly integrated into heterotaxic syndromes. The most frequent liver abnormalities are represented by hepatic congestion, due to hemodynamic changes. Bowel anomalies were essentially malformative with a predominance of hiatal hernias. Kidney anomalies were also malformative, with a horseshoe kidney being the predominant finding.
Table 4 Distribution of infra-diaphragmatic and skeletal findings

\begin{tabular}{lll}
\hline Organ & Anomaly & $\mathbf{n}$ (Patients) \\
\hline Spleen & Asplenia & 10 \\
& Polysplenia & 14 \\
Liver & Congestion & 8 \\
& Hemangioma & 3 \\
& Portal venous thrombosis & 1 \\
& Portal venous gas & 1 \\
\multirow{3}{*}{ Kidney } & Simple hepatic cyst & 1 \\
& Horseshoe kidney & 4 \\
Intestines & Renal agenesis & 2 \\
& Dilation of the urinary tract & 2 \\
& Hiatal hernia & 3 \\
Bone & Common mesentery & 1 \\
& Omphalocele & 1 \\
& Scoliosis & 2 \\
\hline
\end{tabular}

\section{Skeletal anomalies (Table 4; Fig. 4)}

Since the CT coverage excluded the limbs, skeletal findings were solely represented by spine anomalies found in 5 patients. 


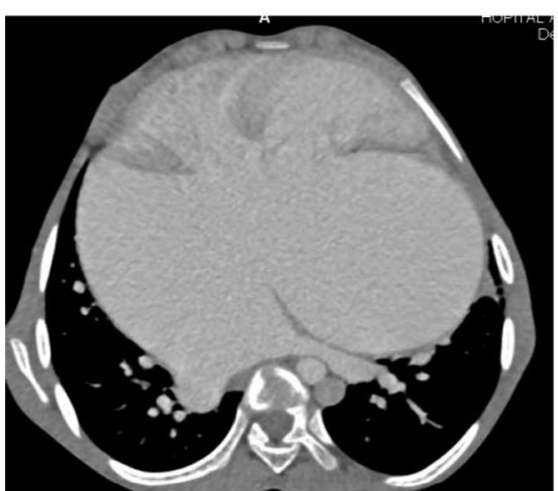

a

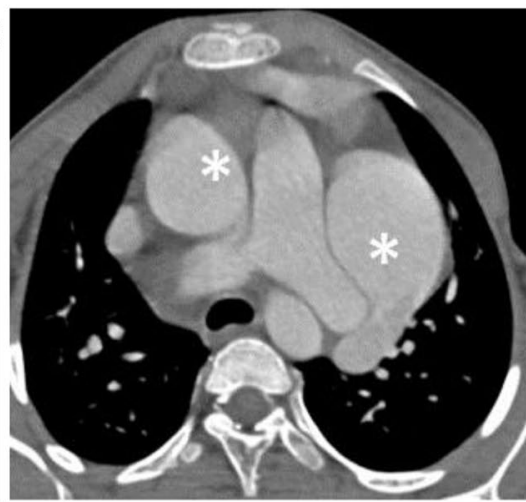

b

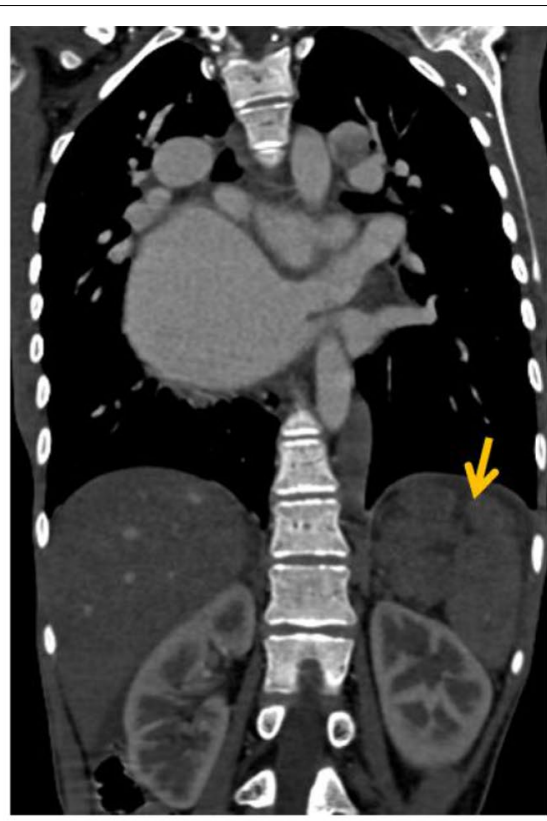

C

Fig. 2 Splenic anomaly. Intra-cardiac total anomalous pulmonary venous return (TAPVR): the 4 pulmonary veins flow directly into the right atrium (a), associated with a levo-isomerism and inter-atrial communication (IAC). There is also a double superior vena cava ( $\left.{ }^{*} \mathbf{b}\right)$ and a polysplenia [yellow arrow $(\mathbf{c})$ ]

\section{Syndromic associations (Table 5; Figs. 5, 6)}

A total of 28 cases of syndromic association were identified, representing approximately $16 \%$ of the patients presenting a congenital heart disease and approximately $27 \%$ of the patients (28 of 105) positive for a non-cardiovascular CT finding. Heterotaxic syndromes were predominant.

\section{Discussion}

The prevalence of non-cardiovascular anomalies is $58 \%$ in our study. In similar studies using CT angiography as the exploration technique, this prevalence is $83 \%$ in the series by Malik et al. [21] and $31.6 \%$ in the series by Rodriguez Martin et al. [20].

There is a moderate variability between these different series. Also, despite the use of a 64 detectors MDCT scanner, Malik et al. [21] and Rodriguez Martin et al. [20] showed a significant difference in the prevalence of the non-cardiovascular findings.

In view of these observations, we deduce a few reflections: we used a 16-detectors MDCT scanner. It is clear that a 64-detectors machine is more efficient. Also, it would be justified to conclude that the higher prevalence of Malik et al. [21], compared to our study, is in a large part, due to the use of a more efficient machine. But this reasoning fails when we compare the results of Malik et al. [21] with those of Rodriguez Martin et al. [20]. This could mean that the performance of the MDCT scanner and the characteristics of the population are both, the key factors determining the prevalence of non-cardiovascular findings. Because the study by Malik et al. [21] was carried out in a reference center specialized in pediatric vascular imaging; therefore, recruiting patients in greater number and severity; whereas our study and that of Rodriguez Martin et al. [20] were carried out in a pediatric imaging department with several activities, some of which are dedicated to pediatric cardiovascular imaging.

Nevertheless, it is obvious that non-cardiovascular findings are frequent. A common point of these three studies is the higher frequency of pulmonary and airway findings observed in $41 \%$ our patients, versus $59 \%$ in Malik et al. series [21] and $16.7 \%$ in that of Rodriguez Martin et al. [20].

Ghadimi Mahani et al. [22] were interested in the prevalence of extra-cardiac abnormalities found on MR angiographies in children with congenital heart disease. They found an overall prevalence of $16.5 \%$ of non-cardiac anomalies with a predominance of abdominal findings (51\% versus $31 \%$ for intrathoracic findings). This difference can be explained by the lower performance of MRI for the study of thoracic structures. 


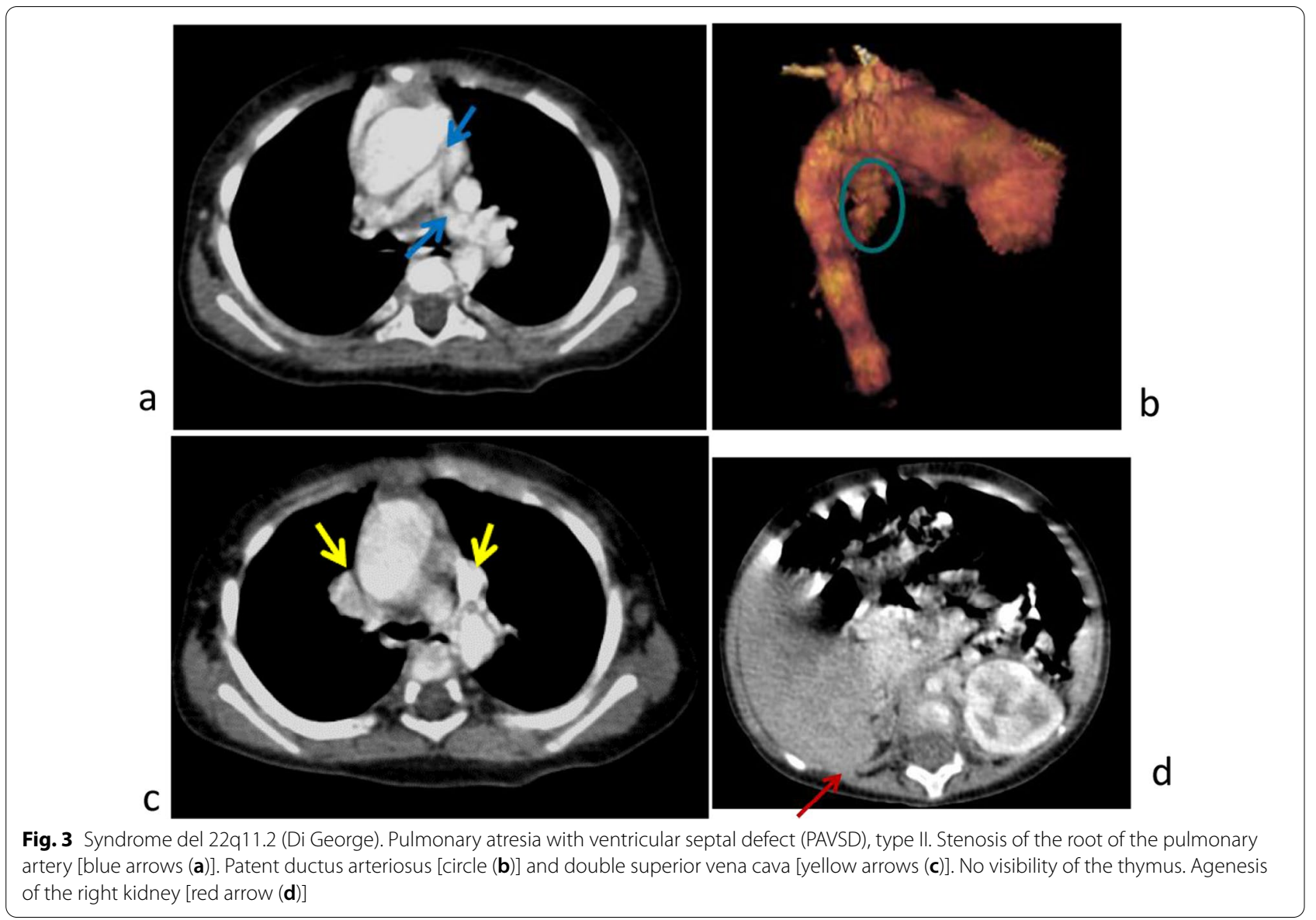

These above-mentioned studies are listed along with ours in (Table 6) showing comparison of their key points.

The importance of these findings varies. Some of them are minor, without significant clinical consequence; others are major, requiring additional investigations or even appropriate immediate treatment. In our series, $49 \%$ of patients with congenital heart disease have at least one significant non-cardiac finding, requiring additional investigations or specific immediate or deferred management. This rate was $74 \%$ in the series of Malik et al. [21], higher than in ours.

This high prevalence of significant non-cardiovascular findings detected on examinations performed for congenital heart disease, requires the radiologist to be vigilant and attentive when analyzing a chest $\mathrm{CT}$ angiography in children. The impact of associated non-cardiovascular lesions on the diagnostic and therapeutic management of the patient can be crucial [19, 21-24].

However, some authors believe that the detection of non-cardiovascular anomalies on cardiac CT angiographies (especially in adults) can be rather a disadvantage, because their detection often leads to additional diagnostic procedures and additional costs without significant improvement of the patient's condition [25, 26].

Such reasoning goes against the goal of our study and many other similar ones, because it could cultivate the laziness of the radiologists as regards to the systematic analysis of all the volume acquired. We also believe that ignoring or not reporting such anomalies is a mistake and could have a negative impact on the patient's outcome.

As a reminder, the data of our study shows that approximately one out of two patients with congenital heart disease have at least one significant non-cardiac finding. Moreover, these significant anomalies can be unexpected, urging the radiologists to look at everything and to report the other findings associated with the congenital heart diseases.

The vast majority of the congenital heart diseases are isolated. However, $20-30 \%$ are the expression of a genetic abnormality fitting into a syndromic framework. Genetic progress has been made in identifying several associated genes in polymalformative genetic syndromes that combine congenital heart disease and extra-cardiac manifestations [27-30]. The knowledge of the 


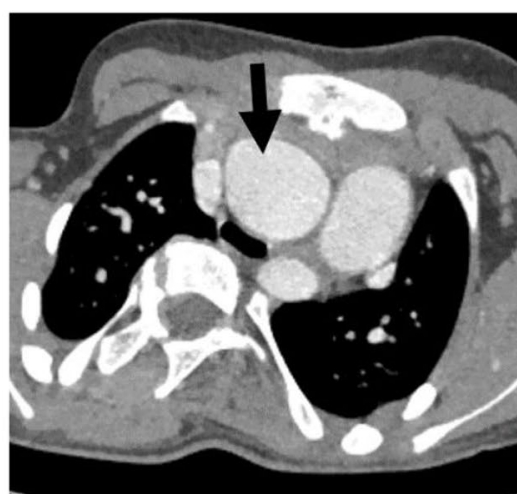

a

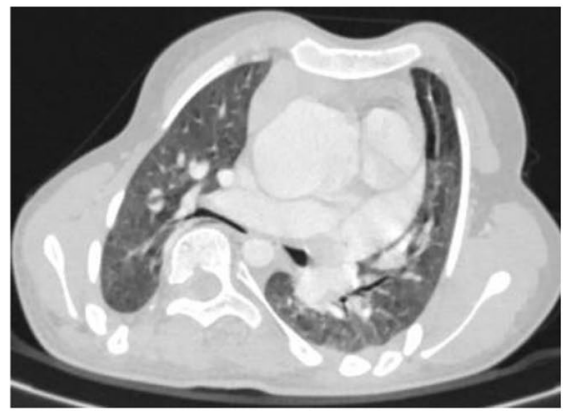

d

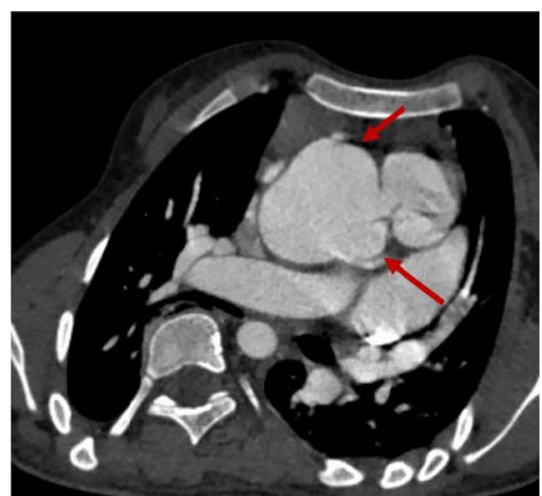

b

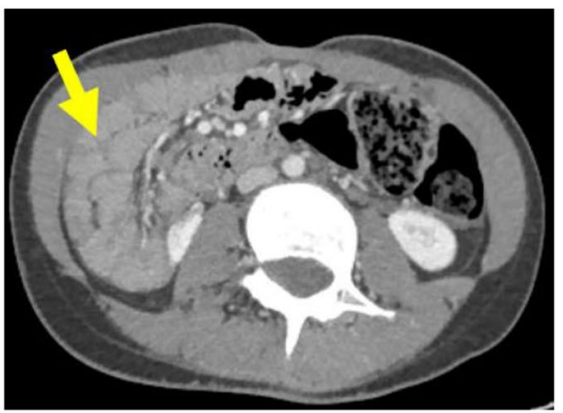

e

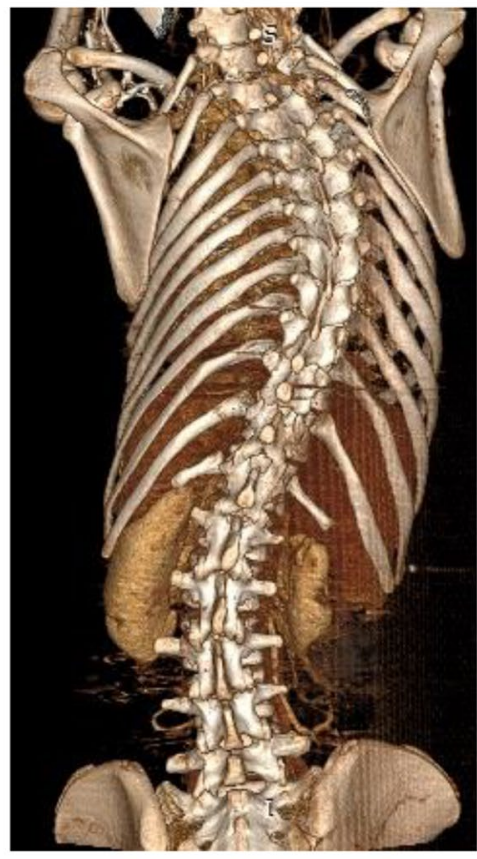

C

Fig. 4 Multiple associated anomalies. 14 years old, Marfan syndrome. Dilation of the aorta [black arrow (a)] and Valsalva sinus aneurysmal dilation [red arrows (b)]. Dilation of the pulmonary artery. Dorsal scoliosis (c) with thoracic deformation and hypoplasia of both lungs (d). Presence of a complete common mesentery; the small intestinal loops being located to the right of the colon [yellow arrow (e)]

Table 5 Distribution of syndromic congenital heart diseases

\begin{tabular}{ll}
\hline Syndromes & $\mathbf{n}=$ Patients \\
\hline Heterotaxic syndromes & 14 \\
Down Syndrome & 4 \\
Williams Beuren & 3 \\
Kartagener & 3 \\
22q11.2 (Di-George) & 2 \\
VACTERL & 1 \\
Marfan & 1 \\
\hline
\end{tabular}

syndromic character of a heart disease requires a systematic investigation of commonly associated extracardiac abnormalities.

In our series, we identified 28 cases of syndromic heart diseases, representing $17 \%$ of all our patients and $27 \%$ of patients with non-cardiovascular findings. These syndromic heart diseases have a prevalence of $22 \%$ in the series of Malik et al. [21] among which half had genetic confirmation only after radiological suspicion. This again underlines the importance of detecting and reporting these findings.

\section{Critical analysis of the study}

The first bias is the retrospective nature of the study. There is an intrinsic incomplete nature of the recruitment of patients and of the data collection because, for example, if a desired data is missing from the report, it is considered to be really absent when it could have been present if the study had been prospective and we had systematically planned to search for particular anomalies.

Also, given the retrospective nature of our study, there are chances of lack of adequate monitoring of the evolution of non-cardiovascular abnormalities in order to effectively weigh their importance in the management of the patient.

The data collected depends on the skills of a single reader. It is obvious that adding a second one would minimize the risk of omission and add more value to our observations. Despite this, we are confident in our excellent diagnostic ability in congenital heart diseases.

The separation between significant and non-significant findings can sometimes be delicate. In addition, a direct attribution of an anatomical abnormality to a clinical manifestation is not obvious because the pathophysiological implications can be complex. 

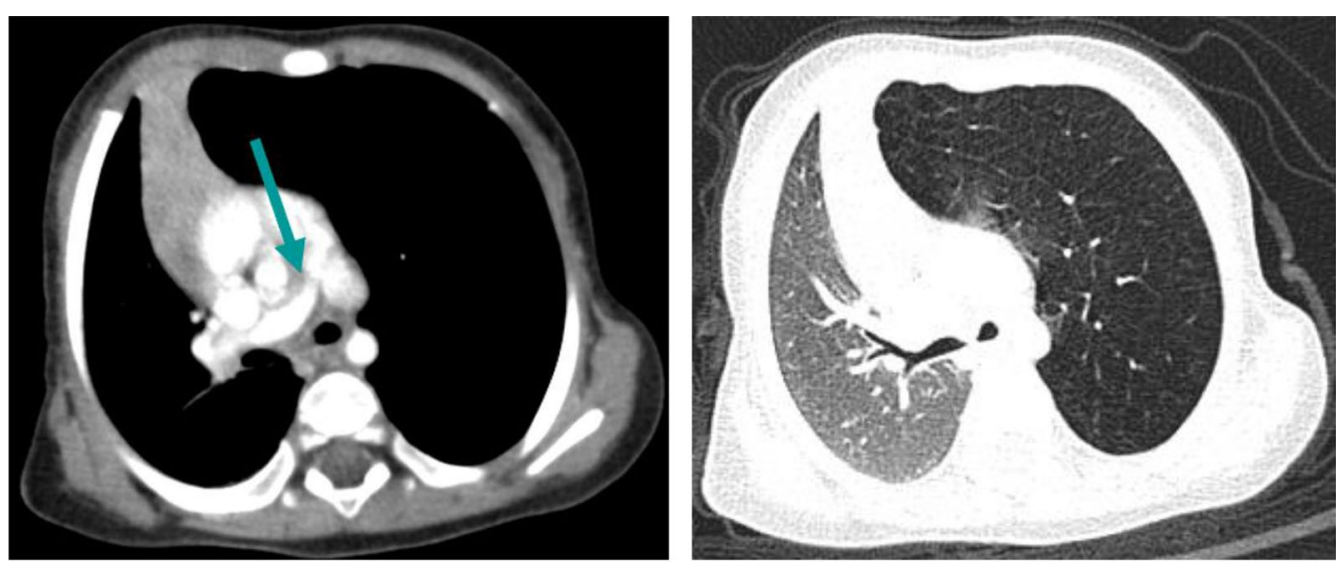

a

b
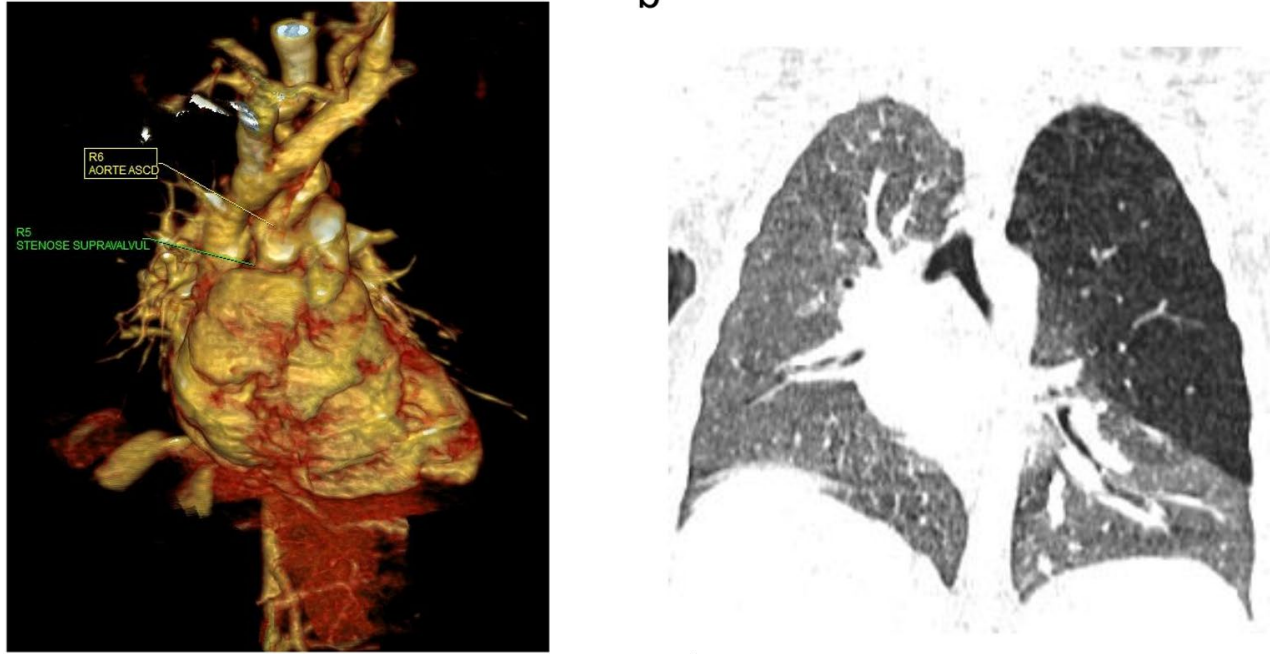

C

d

Fig. 5 Williams Beuren syndrome. Supravalvular aortic stenosis associated with a tight stenosis of the root of the right pulmonary artery [arrows (a, c)]. Congenital lobar emphysema of the superior pulmonary left lobe (b, d).

Last but not least, we used a single source 16-detectors MDCT scanner. It obvious that a better machine would have grant us a better quality of the data.

Nevertheless, despite these limitations, our results are usable. This study allowed us to make a real observation of the prevalence, significance and polymorphism of these non-cardiovascular findings associated with congenital heart disease in children explored by $\mathrm{CT}$ angiography.

It should lead us to place more emphasis on the systematic analysis of the acquired volume, in order to detect the maximum associated lesions, since they can have major impact in the management of the patients.

\section{Conclusion}

Non-cardiovascular findings are frequent on chest $\mathrm{CT}$ angiography in children with congenital heart disease. The data of our series showed that almost $2 / 3$ of these children with congenital heart disease have one or several non-cardiovascular anomalies, of which up to $50 \%$ can be significant requiring additional investigations and / or contributing to the patient's symptoms; therefore, requiring immediate or deferred appropriate management. Their detection is essential for the optimal management of these patients.

These observations underline the importance of a systematic analysis of all the acquired volume of chest $\mathrm{CT}$ angiographies. 

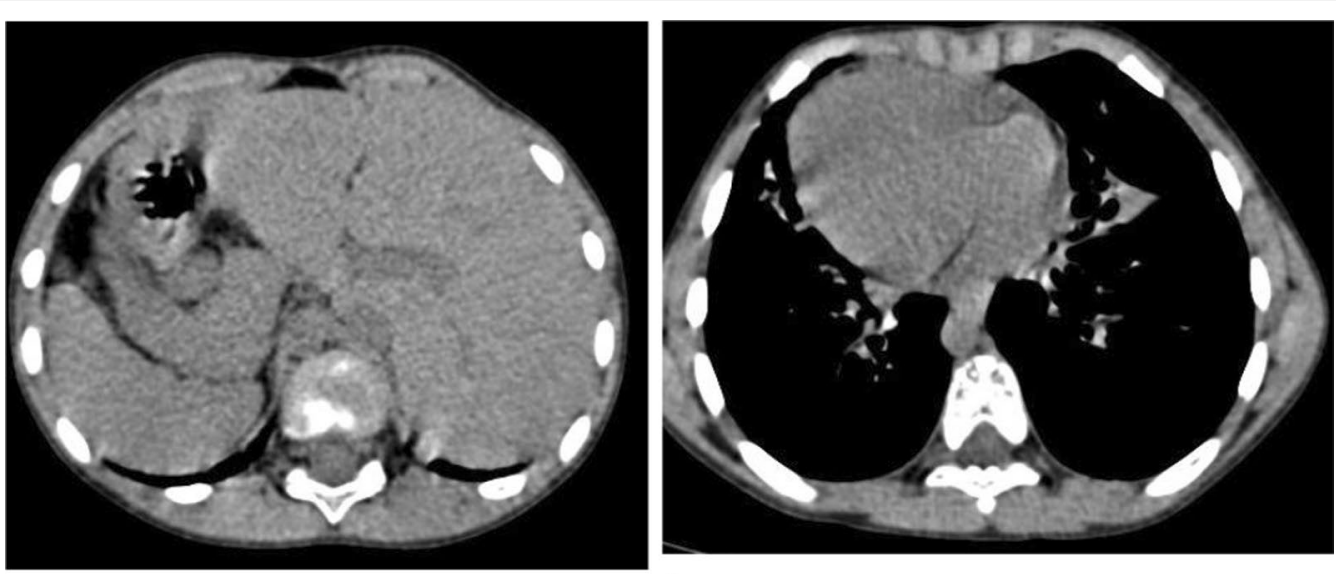

a

b

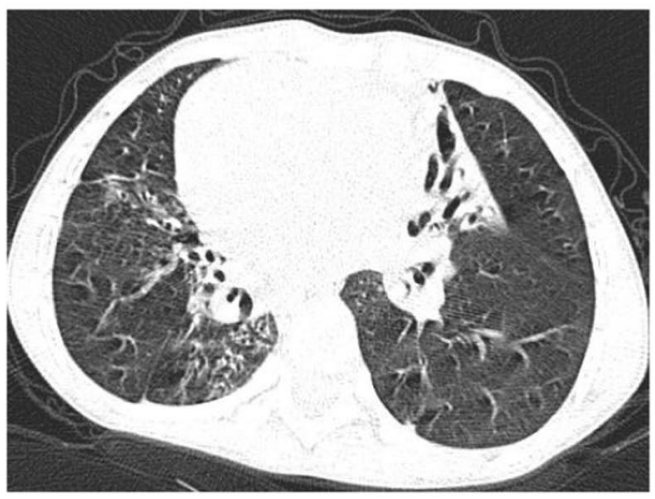

C

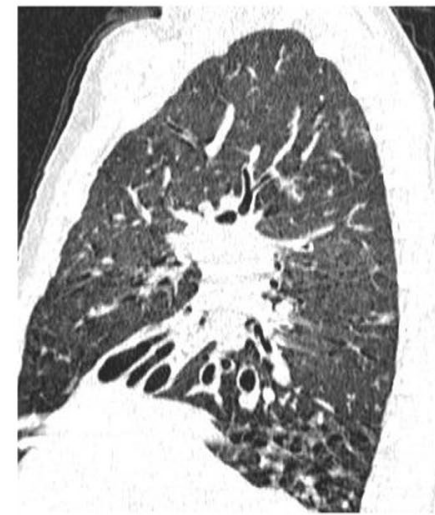

d

Fig. 6 Kartagener syndrome. Situs inversus (a, b). Bilateral bronchiectasis (c, d)

Table 6 Comparative listing of the different studies

\begin{tabular}{|c|c|c|c|c|c|c|c|}
\hline Study & Imaging technic & Period & Type & $\begin{array}{l}\text { Number of } \\
\text { patients } \\
\text { (n) }\end{array}$ & $\begin{array}{l}\text { Prevalence of } \\
\text { non-cardiovascular } \\
\text { findings } \\
(\%)\end{array}$ & $\begin{array}{l}\text { Significant non- } \\
\text { cardiovascular } \\
\text { findings } \\
(\%)\end{array}$ & $\begin{array}{l}\text { Predominant } \\
\text { findings }\end{array}$ \\
\hline Malik et al. [21] & CTA & 2 years and 4 months & Retrospective & 300 & 83 & 73.7 & Lungs \\
\hline $\begin{array}{l}\text { Ghadimi Mahani et al. } \\
{[22]}\end{array}$ & MRA & $\begin{array}{l}4 \text { years and } 10 \\
\text { months }\end{array}$ & Retrospective & 740 & 16.5 & 2.6 & Abdominal \\
\hline Rodriguez Martin [20] & CTA & 5 years & Retrospective & 222 & 31.6 & 22.7 & Lungs \\
\hline Our study & CTA & 4 years and 3 months & Retrospective & 180 & 58 & 49 & Lungs \\
\hline
\end{tabular}

\section{Abbreviations}

ALARA: As low as reasonably achievable; MRI: Magnetic resonance imaging; CT: Computed tomography; MIP: Maximum intensity projection; MinIP. Minimum intensity projection; VRT: Volume rendering technic; 3D: Threedimensions; MDCT: Multidetector-row computed tomography.

\section{Acknowledgements}

None.

\section{Authors' contributions}

YEN, SRAI and LC equally contributed to this work. YEN: conception, design of the work, acquisition, analysis, interpretation of data, manuscript writing editing of manuscript. SRAl: conception, design of the work, acquisition, analysis, interpretation of data, manuscript writing, editing of manuscript. LC: conception, design of the work, acquisition, analysis, interpretation of data, manuscript writing, editing and final review of the manuscript. All authors read and approved the final manuscript. 


\section{Funding}

This manuscript is not supported by any grants.

\section{Availability of data and materials}

The datasets used and/or analyzed during the current study are available from the corresponding author on reasonable request.

\section{Declarations}

\section{Ethics approval and consent to participate}

This study was approved by the local Ethical Committee of our institution (Local Ethical committee of the Children's Hospital-Centre HospitaloUniversiataire Ibn Sina of Rabat-Morocco) and a written informed consent was waived owing to the retrospective, observational, and anonymous nature of this study. The methods were carried out in accordance with the relevant guidelines and regulations.

\section{Consent for publication}

This study was approved by the local Ethical Committee of our institution (Local Ethical committee of the Children's Hospital-Centre Hospitalo-Universiataire Ibn Sina of Rabat-Morocco).

\section{Competing interests}

The authors declare that they have no competing interests.

Received: 9 September 2021 Accepted: 19 January 2022 Published online: 22 January 2022

\section{References}

1. Reller MD, Strickland MJ, Riehle-Colarusso T, Mahle WT, Correa A. Prevalence of congenital heart defects in metropolitan Atlanta, 1998-2005. J Pediatr. 2008;153(6):807-13. https://doi.org/10.1016/j.jpeds.2008.05.059.

2. Hoffman JI, Kaplan S. The incidence of congenital heart disease. J Am Coll Cardiol. 2002;39(12):1890-900.

3. Botto LD, Correa A, Erickson JD. Racial and temporal variations in the prevalence of heart defects. Pediatrics. 2001;107(3):E32.

4. Hellinger JC, Pena A, Poon M, Chan FP, Epelman M. Pediatric computed tomographic angiography: imaging the cardiovascular system gently. Radiol Clin North Am. 2010;48(2):439-67, x. https://doi.org/10.1016/j.rcl. 2010.03.005.

5. Koestenberger M, Friedberg MK, Ravekes W, Nestaas E, Hansmann G. Non-invasive imaging for congenital heart disease: recent innovations in transthoracic echocardiography. J Clin Exp Cardiolog. 2012;Suppl 8:2.

6. Han BK, Lesser JR. CT imaging in congenital heart disease: an approach to imaging and interpreting complex lesions after surgical intervention for tetralogy of Fallot, transposition of the great arteries, and single ventricle heart disease. J Cardiovasc Comput Tomogr. 2013;7(6):338-53. https:// doi.org/10.1016/j.jcct.2013.10.003.

7. Kulkarni A, Hsu HH, Ou P, Kutty S. Computed tomography in congenital heart disease: clinical applications and technical considerations. Echocardiography. 2016;33(4):629-40. https://doi.org/10.1111/echo.13147.

8. Han BK, Overman DM, Grant K, Rosenthal K, Rutten-Ramos S, Cook D, Lesser JR. Non-sedated, free breathing cardiac CT for evaluation of complex congenital heart disease in neonates. J Cardiovasc Comput Tomogr. 2013;7(6):354-60. https://doi.org/10.1016/j.jcct.2013.11.006.

9. Dillman JR, Hernandez RJ. Role of CT in the evaluation of congenital cardiovascular disease in children. AJR Am J Roentgenol. 2009;192(5):121931. https://doi.org/10.2214/AJR.09.2382.

10. Goo HW. Cardiac MDCT in children: CT technology overview and interpretation. Radiol Clin North Am. 2011;49(5):997-1010. https://doi.org/10. 1016/j.rcl.2011.06.001

11. Greenberg SB, Bhutta S, Braswell L, Chan F. Computed tomography angiography in children with cardiovascular disease: low dose techniques and image quality. Int J Cardiovasc Imaging. 2012;28(1):163-70. https:// doi.org/10.1007/s10554-010-9782-1.

12. Haller S, Kaiser C, Buser P, Bongartz G, Bremerich J. Coronary artery imaging with contrast-enhanced MDCT: extracardiac findings. AJR Am J Roentgenol. 2006;187(1):105-10.
13. Onuma Y, Tanabe K, Nakazawa G, Aoki J, Nakajima H, Ibukuro K, Hara K. Noncardiac findings in cardiac imaging with multidetector computed tomography. J Am Coll Cardiol. 2006;48(2):402-6.

14. Chia PL, Kaw G, Wansaicheong G, Ho KT. Prevalence of non-cardiac findings in a large series of patients undergoing cardiac multi-detector computed tomography scans. Int J Cardiovasc Imaging. 2009;25(5):537-43. https://doi.org/10.1007/s10554-009-9455-0.

15. Dewey M, Schnapauff D, Teige F, Hamm B. Non-cardiac findings on coronary computed tomography and magnetic resonance imaging. Eur Radiol. 2007;17(8):2038-43.

16. Sohns C, Sossalla S, Vollmann D, et al. Extracardiacfindings by 64 -multidetector computed tomography in patients with symptomatic atrial fibrillation prior to pulmonal vein isolation. Int J Cardiovasc Imaging. 2011;27:127. https://doi.org/10.1007/s10554-010-9653-9.

17. Bendix K, Jensen JM, Poulsen S, et al. Coronary dual source multi detector computed tomography in patients suspected of coronary artery disease: prevalence of incidental extra-cardiac findings. Eur J Radiol. 2011;80:10914. https://doi.org/10.1016/j.ejrad.2010.05.024.

18. May CW, Mansfield WT, Landes AB, Moran AM. Prevalence of noncardiac findings in patients undergoing cardiac magnetic resonance imaging. Sci World J. 2012;2012: 474582. https://doi.org/10.1100/2012/474582.

19. Buckens CF, Verkooijen HM, Gondrie MJ, Jairam P, Mali WP, van der Graaf Y. Unrequested findings on cardiac computed tomography: looking beyond the heart. PLOS ONE. 2012;7(4): e32184. https://doi.org/10.1371/ journal.pone.0032184.

20. J. I. Rodríguez Martín, M. Bret Zurita, E. Cuesta López, L. Polo López, A. Cartón Sánchez, F. Gutiérrez-Larraya Aguado; Madrid/ES. Unexpected findings in cardiac imaging of pediatric patients with congenital heart disease using 64 detector computed tomography. ECR 2019/ C-2538. https://doi.org/10.26044/ecr2019/C-2538

21. Malik A, Hellinger JC, Servaes S, et al. Prevalence of non-cardiovascular findings on CT angiography in children with congenital heart disease. Pediatr Radiol. 2017;47:267. https://doi.org/10.1007/s00247-016-3742-4.

22. Ghadimi Mahani M, Morani AC, Lu JC, et al. Non-cardiovascular findings in clinical cardiovascular magnetic resonance imaging in children. Pediatr Radiol. 2016;46:473. https://doi.org/10.1007/s00247-015-3512-8.

23. Sohns C, Sossalla S, Vollmann D, et al. Extra cardiac findings by 64-multidetector computed tomography in patients with symptomatic atrial fibrillation prior to pulmonal vein isolation. Int J Cardiovasc Imaging. 2011;27:127. https://doi.org/10.1007/s10554-010-9653-9.

24. Gil BN, Ran K, Tamar G, Shmuell F, Eli A. Prevalence of significant noncardiac findings on coronary multidetector computed tomography angiography in asymptomatic patients. J Comput Assist Tomogr. 2007;31 (1):1-4. https://doi.org/10.1097/01.rct.0000233125.83184.33.

25. Budoff MJ, Fischer H, Gopal A. Incidental findings with cardiac CT evaluation: should we read beyond the heart? Catheter Cardiovasc Interv. 2006;68(6):965-73. https://doi.org/10.1002/ccd.20924.

26. Budoff MJ, Gopal A. Incidental findings on cardiac computed tomography. Should we look? J Cardiovasc Comput Tomogr. 2007;1 (2):97-105. https://doi.org/10.1016/j.jcct.2007.04.002.

27. Calcagni G, Unolt M, Digilio MC, Baban A, Versacci P, Tartaglia M, Baldini A, Marino B. Congenital heart disease and genetic syndromes: new insights into molecular mechanisms. Expert Rev Mol Diagn. 2017;17(9):861-70. https://doi.org/10.1080/14737159.2017.1360766.

28. Ko JM. Genetic Syndromes associated with Congenital Heart Disease. Korean Circ J. 2015;45(5):357-61.

29. Mani A, Alizadehasl A. Syndromic congenital heart diseases. In: Sadeghpour A, Kyavar M, Alizadehasl A, editors. Comprehensive approach to adult congenital heart disease. London: Springer; 2014. p. 65-9. https:// doi.org/10.1007/978-1-4471-6383-1_10.

30. Fahed AC, Gelb BD, Seidman JG, Seidman CE. Genetics of congenital heart disease: the glass half empty. Circ Res. 2013;112(4):707-20. https:// doi.org/10.1161/CIRCRESAHA.112.300853.

\section{Publisher's Note}

Springer Nature remains neutral with regard to jurisdictional claims in published maps and institutional affiliations. 